\title{
E. coli 0157 on Scottish cattle farms: Evidence of local spread and persistence using repeat cross-sectional data
}

\author{
Liam J Herbert ${ }^{1}$, Leila Vali², Deborah V Hoyle ${ }^{1}$, Giles Innocent ${ }^{3}$, lain J McKendrick ${ }^{3}$, Michael C Pearce ${ }^{4}$, \\ Dominic Mellor ${ }^{5}$, Thibaud Porphyre ${ }^{1}$, Mary Locking ${ }^{6}$, Lesley Allison ${ }^{7}$, Mary Hanson $^{7}$, Louise Matthews ${ }^{8}$, \\ George J Gunn ${ }^{9}$, Mark EJ Woolhouse ${ }^{1}$ and Margo E Chase-Topping ${ }^{1 *}$
}

\begin{abstract}
Background: Escherichia coli (E. coli) $\mathrm{O} 157$ is a virulent zoonotic strain of enterohaemorrhagic E. coli. In Scotland (1998-2008) the annual reported rate of human infection is 4.4 per 100,000 population which is consistently higher than other regions of the UK and abroad. Cattle are the primary reservoir. Thus understanding infection dynamics in cattle is paramount to reducing human infections.

A large database was created for farms sampled in two cross-sectional surveys carried out in Scotland (1998 - 2004).

A statistical model was generated to identify risk factors for the presence of E. coli 0157 on farms. Specific hypotheses were tested regarding the presence of E. coli $\mathrm{O} 157$ on local farms and the farms previous status. Pulsed-field gel electrophoresis (PFGE) profiles were further examined to ascertain whether local spread or persistence of strains could be inferred.
\end{abstract}

Results: The presence of an E. coli $\mathrm{O} 157$ positive local farm (average distance: 5.96km) in the Highlands, North East and South West, farm size and the number of cattle moved onto the farm 8 weeks prior to sampling were significant risk factors for the presence of E. coli 0157 on farms. Previous status of a farm was not a significant predictor of current status $(p=0.398)$. Farms within the same sampling cluster were significantly more likely to be the same PFGE type $(p<0.001)$, implicating spread of strains between local farms. Isolates with identical PFGE types were observed to persist across the two surveys, including 3 that were identified on the same farm, suggesting an environmental reservoir. PFGE types that were persistent were more likely to have been observed in human clinical infections in Scotland $(p<0.001)$ from the same time frame.

Conclusions: The results of this study demonstrate the spread of E. coli O157 between local farms and highlight the potential link between persistent cattle strains and human clinical infections in Scotland. This novel insight into the epidemiology of Scottish E. coli O157 paves the way for future research into the mechanisms of transmission which should help with the design of control measures to reduce E. coli 0157 from livestock-related sources.

Keywords: E. coli O157, Epidemiology, Risk factor, Transmission, Persistence, PFGE

\section{Background}

Escherichia coli (E. coli) O157 is a strain of enterohaemorrhagic E. coli (EHEC), also classified as a strain of Shigatoxin producing $E$. coli (STEC) or verocytotoxin producing E. coli (VTEC). Since 1982, E. coli O157 has been recognized as an important zoonotic gastrointestinal pathogen of

\footnotetext{
* Correspondence: margo.chase@ed.ac.uk

'Centre for Immunity, Infection and Evolution, University of Edinburgh, King's Buildings, Edinburgh, UK

Full list of author information is available at the end of the article
}

humans. Although the reported incidence is often low, E. coli $\mathrm{O} 157$ infections are frequently publicized due to large outbreaks and the severity of the illness that it causes, particularly in children and the elderly. Primarily transmitted by the faecal-oral route, infection can arise from animal to human contact, both direct and indirect, human-to-human contact or by foodborne transmission [1].

Escherichia coli $\mathrm{O} 157$ is the most common reported EHEC serotype in the UK and most countries globally. More than 50 countries have reported cases of human

\section{Ciomed Central}


infection with E. coli O157, across the 6 inhabited continents [2]. The highest annual incidences of human infection with $E$. coli O157 during the last two decades have been reported in parts of Canada, the United States, Japan and Scotland [3,4]. In Scotland, the mean reported incidence is 4.4 cases per 100,000 population per year (1999-2008) which is consistently higher than which is observed in most other regions of the UK and the world [5].

Cattle are the primary reservoir of E. coli O157, and these animals typically have transient asymptomatic infection [6]. Farm-level prevalence of E. coli O157 on Scottish beef rearing farms has been estimated at around $21 \%$ across farms widely distributed throughout Scotland [7] although a recent study proposed that while $\sim 20 \%$ of farms are E. coli $\mathrm{O} 157$ positive at any one time, in a year $>80 \%$ of farms will be infected [6]. Widespread agricultural contamination produces a plethora of public health risks. Precautionary principle now requires that all farms in Scotland should be considered to be contaminated [8]. Hence there is a need to minimize the risk of human E. coli O157 infection from livestock. This can be achieved through further understanding of transmission.

Natural transmission of E. coli O157 between cattle is thought to occur largely through the faecal-oral route, although this may occur indirectly via an environmental reservoir [9]. Persistence and spreading of E. coli O157 within farms can be influenced by strain type, duration of shedding, prevalence, magnitude of shedding by individual animals and bacterial survival and growth in the farm environment [10]. Faecal shedding in individual cattle is mainly transient [11] and E. coli $\mathrm{O} 157$ prevalence is known to be highly skewed [12], most cattle groups test negative for the pathogen, but a small proportion shed high numbers of E. coli O157 (i.e. supershedders). Cattle that excrete high numbers of bacteria can be expected to pose a greater risk of infection to other cattle and humans than those excreting bacteria in low numbers [2].

Persistence of E. coli $\mathrm{O} 157$ is commonly defined at individual animal level owing to the transient nature of cattle carriage. There is previously published information about persistence in different cattle production systems [13-18]. Individual E. coli O157 strains have been isolated for as long as 2 years from dairy herds [14], for as long as 10 months on cattle ranges [16] and over the entire feeding period on cattle feedlots [17]. Liebana et al. [18] examined 11 cattle farms across England and Wales and found that most strains (identified using pulsed field gel electrophoresis (PFGE)) were found only on individual farms but some were found on multiple farms. In another longitudinal study of 9 epidemiologically unrelated farms dispersed across England and Wales Liebana et al. [15] found that some clones can be isolated from the same farm over a period of at least 17 months and from the same animal for a period of at least 7 months. To date there has been no equivalent study in Scotland that has examined local spread and persistence. As such information on farm level persistence of E. coli O157 populations in Scotland are less understood.

The objectives of this research were twofold. First to examine the risk factors for the presence of E. coli O157 on Scottish farms specifically testing hypotheses regarding local spread and the previous status of a farm. Previous research using data generated from survey 2 in this study failed to provide evidence of local spread [6]. However, the associations within that study were based on the spatial clustering of farms with no direct knowledge of the status of local farms in the area. Second, use PFGE profiles to look for direct evidence of local spread and the presence of persistent strains. To our knowledge this is the first study to examine these issues using data from repeated cross sectional surveys and we aim to expand the available evidence into the factors that influence farm-level carriage and persistence of E. coli O157.

\section{Results}

\section{Agreement of farm status at both time points}

The majority of farms (63\%) were negative in both surveys $(n=283 / 447)$ (Table 1$)$. Thirty-one percent of the farms changed status either positive to negative (17.2\%, $\mathrm{n}=77 / 447)$ or negative to positive $(13.4 \%, \mathrm{n}=60 / 447)$. Only $6 \%$ of the farms ( $\mathrm{n}=27 / 447)$ were positive for $E$. coli $\mathrm{O} 157$ in both surveys. There was no change in the proportion of positive farms between the two surveys (McNemar's test, $\mathrm{p}=0.171$ ).

\section{Analysis of risk factors: single variate and multiple variate results}

Fourty-nine variables were screened as possible risk factors for this study (Additional file 1: Table S1). Twentytwo variables which had a $\mathrm{p}$ value of $<0.2$ (Table 2) were carried forward to multiple variate analysis. Of the 22 variables, only 2 remained significant as single variables in the multiple variate analysis: the total number of cattle on the farm $(\mathrm{p}<0.001)$ and the number of movements onto the farm in the 8 weeks preceding the

Table 1 Farm E. coli 0157 status

\begin{tabular}{lcccc}
\hline & & \multicolumn{2}{c}{ Survey 2 } & \\
\cline { 3 - 4 } & & Positive & Negative & Total \\
\hline Survey 1 & Positive & 27 & 77 & 104 \\
& Negative & 60 & 283 & 343 \\
& Total & 87 & 360 & 447 \\
\hline
\end{tabular}

Status of Scottish farms (positive or negative) during Survey 1 (SEERAD, 19982000) and Survey 2 (IPRAVE, 2002-2004). A positive farm was one in which at least one faecal pat tested positive by Immuno-magnetic separation (IMS) for the presence of E. coli 0157 . 
Table 2 Single variate analysis of risk factors

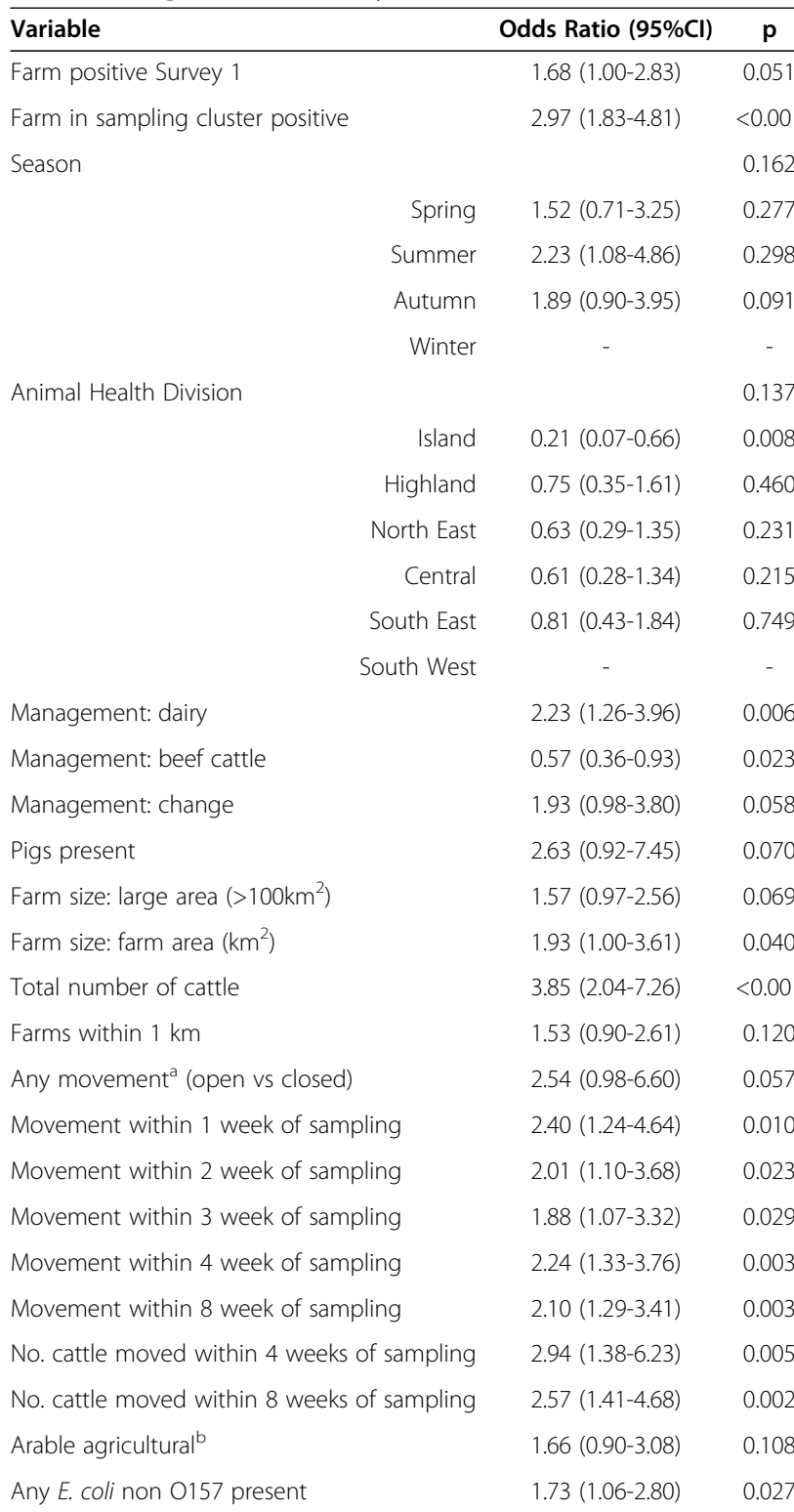

Variables identified from single variate logistic regression models for the presence of E. coli 0157 on the 447 Scottish farms that were sampled in both Survey 1 (SEERAD, 1998-2000) and Survey 2 (IPRAVE, 2002-2004).

${ }^{a}$ Movement (as defined by Cattle Tracing System (CTS) [20]) is deemed to have occurred when an animal is moved on to a specific farm or between herds.

${ }^{\mathrm{b}}$ Arable agriculture (Land Capability for Agriculture class 1-3.1). Prime agricultural land capable for being used to produce a wide range of crops. Favourable climate; slopes are no greater than 7 degrees; soils are at least $45 \mathrm{~cm}$ deep; imperfectly drained [21].

sampling in Survey $2(\mathrm{p}=0.035)$ were significant risk factors for farm carriage of E. coli O157 (Table 3). The previous status of the farm in Survey 1 was not a significant predictor $(p=0.398)$ of whether or not a farm was positive in Survey 2. Cluster positivity, i.e. another (local) farm in the Survey 2 sampling cluster being positive for E. coli $\mathrm{O} 157$ was significant both as a single variable $(\mathrm{p}<$ 0.001) and in an interaction with Animal Health District
Table 3 Multiple variate analysis of risk factors

\begin{tabular}{|c|c|c|c|}
\hline Predictor & Estimate & SE & $\mathrm{p}$ \\
\hline Farm positive in Survey 1 & 0.264 & 0.3120 & 0.398 \\
\hline Season & & & 0.680 \\
\hline Spring & 0.132 & 0.4293 & 0.758 \\
\hline Summer & 0.478 & 0.4198 & 0.254 \\
\hline Autumn & 0.220 & 0.4218 & 0.603 \\
\hline Winter & - & - & - \\
\hline Farm in sampling cluster positive & 1.719 & 0.6085 & $<0.001$ \\
\hline Animal Health Division (AHD) & & & 0.686 \\
\hline Island & -0.907 & 0.8698 & 0.298 \\
\hline Highland & 0.128 & 0.6842 & 0.852 \\
\hline North East & -0.553 & 0.7340 & 0.451 \\
\hline Central & 0.555 & 0.6139 & 0.367 \\
\hline South East & 0.827 & 0.5956 & 0.166 \\
\hline South West & - & - & - \\
\hline Farm in sampling cluster positive*AHD & & & 0.004 \\
\hline Farm in sampling cluster positive in Islands & 2.215 & 1.1426 & 0.053 \\
\hline Farm in sampling cluster positive in Highland & 1.801 & 0.6558 & 0.006 \\
\hline Farm in sampling cluster positive in North East & 2.441 & 0.7202 & 0.001 \\
\hline Farm in sampling cluster positive in Central & -0.373 & 0.6894 & 0.589 \\
\hline Farm in sampling cluster positive in South East & -0.417 & 0.5948 & 0.484 \\
\hline Farm in sampling cluster positive in South West & 1.719 & 0.6085 & 0.005 \\
\hline Total number of cattle ${ }^{a}$ & 1.401 & 0.3916 & $<0.001$ \\
\hline No. of movements onto farm in last 8 weeks ${ }^{a}$ & 0.774 & 0.3658 & 0.035 \\
\hline Overall $O R^{b}$ & \multicolumn{3}{|c|}{$2.61(0.79)$} \\
\hline
\end{tabular}

Results of the logistic regression model of risk factors for the presence of $E$. coli $\mathrm{O} 157$ on the 447 Scottish farms that were sampled in both Survey 1 (SEERAD, 1998-2000) and Survey 2 (IPRAVE, 2002-2004). Overall OR gives empirical estimate of odds ratio for the entire model.

${ }^{a} \log _{10}$ transformed.

${ }^{\mathrm{b}}$ Mean (SD).

(AHD) $(\mathrm{p}=0.004)$. It appears that having a local farm that is positive may only be considered a risk factor in certain AHDs including the Highlands, North East and South West of Scotland. The overall odds ratio for this model was 2.61, with a standard deviation of 0.79 . The model shows no evidence of lack of fit (Hosmer-Lemeshow goodness-of-fit chi square $=6.1825 \mathrm{df}=8 \mathrm{p}=$ 0.627). The discriminatory power of the model, represented by the AUC statistic was 0.786 . An AUC between 0.7-0.8 gives a fair discriminatory power [19].

\section{Local spread and persistance}

The 87 positive farms from Survey 2 (Table 1) were distributed within 65 of the 161 sampling clusters, and from these farms 500 separate E. coli $\mathrm{O} 157$ isolates were recovered. There were 21 clusters in which two or more farms were positive; 20 had 2 farms positive, 1 cluster had all 3 farms positive. These data were used to test the hypothesis that E. coli $\mathrm{O} 157$ could spread between farms 
in close proximity. Isolates sharing the same phage type (PT) on different farms were found in 10 of the 21 clusters $(48 \%)$. This was not significantly different from random $(10 / 21$ vs $3901 / 10,000 ; p=0.15)$. PT $21 / 28$ was the phage type identified in 9 of the $10(90 \%)$ clusters where the phage type was the same. PT21/28 was the most common phage type identified in this study $(\mathrm{n}=252 /$ $500,50 \%)$. As there are so few PTs ( $\mathrm{n}=12$ different types), by random many are the same. Isolates with indistinguishable PFGE profiles were detected in 4 of the 21 (19\%) sampling clusters. This is significantly different from random (4/21 vs 151/10,000; p < 0.001).

Across the 447 farms sampled in both surveys, 139 different PFGE profiles were identified of which $12(8.6 \%)$ were common to both surveys, but not necessarily common to a particular farm. Figure 1 shows the distribution of the 139 PFGE profiles across the two surveys. The first 12 PFGE profiles are those that are present in both surveys (i.e. persistent). Of the 12 persistent PFGE profiles, only 2 (PFGE designated profiles 2 and 3 ) are well represented in both surveys. Diversity of the PFGE profiles (inset Figure 1) was significantly higher for Survey 2 (the IPRAVE survey) by every measure examined (species richness (SR), Shannon entrophy (SE), Simpson diversity (SD) and Berger Parker (BP)). PFGE profiles marked with an asterisk represent those that were observed in humans (L. Vali unpublished data). Persistent strains were more likely than chance to be those that were identified in humans (Chi-square test, $\mathrm{p}<0.001$ ). Six of the 12 strains $(50 \%)$ identified as present in both surveys were also observed in human clinical infections.
Although persistent strains were identified they were not necessarily identified on the same farm. Of the 27 farms that were positive for E. coli O157 in both Survey 1 and Survey 2 (Table 1), 24 had PFGE profiles for isolates from each survey. The number of farms with the same PFGE profile present on a farm at the two time points ( 2 farms out of 24 ) was compared to a large bootstrap sample of all farms in the two surveys where two different farms were randomly paired (444 out of $10,000)$. These proportions were not significantly different $(\mathrm{p}=0.090)$.

\section{Discussion}

To our knowledge, the surveys examined in this study represent the only reported systematic national surveys of bovine E. coli $\mathrm{O} 157$ shedding and present a valuable opportunity to simultaneously examine factors influencing cattle carriage and persistence of E. coli $\mathrm{O} 157$ at farm level. Although the repeated cross-sectional design has been employed for analysis of E. coli O157 previously [22], we know of no other paper on the topic with the additional benefit of molecular analysis. Furthermore, the use of PFGE data in this study is also advantageous as PFGE methods have been used previously to show persistent and local spread in other E. coli $\mathrm{O} 157$ research $[17,23,24]$.

This study analyses a very large database with risk factors representing farm size, composition and clustering, farm management, feed, infection status, and landscape type (Additional file 1: Table S1). Despite the comprehensive
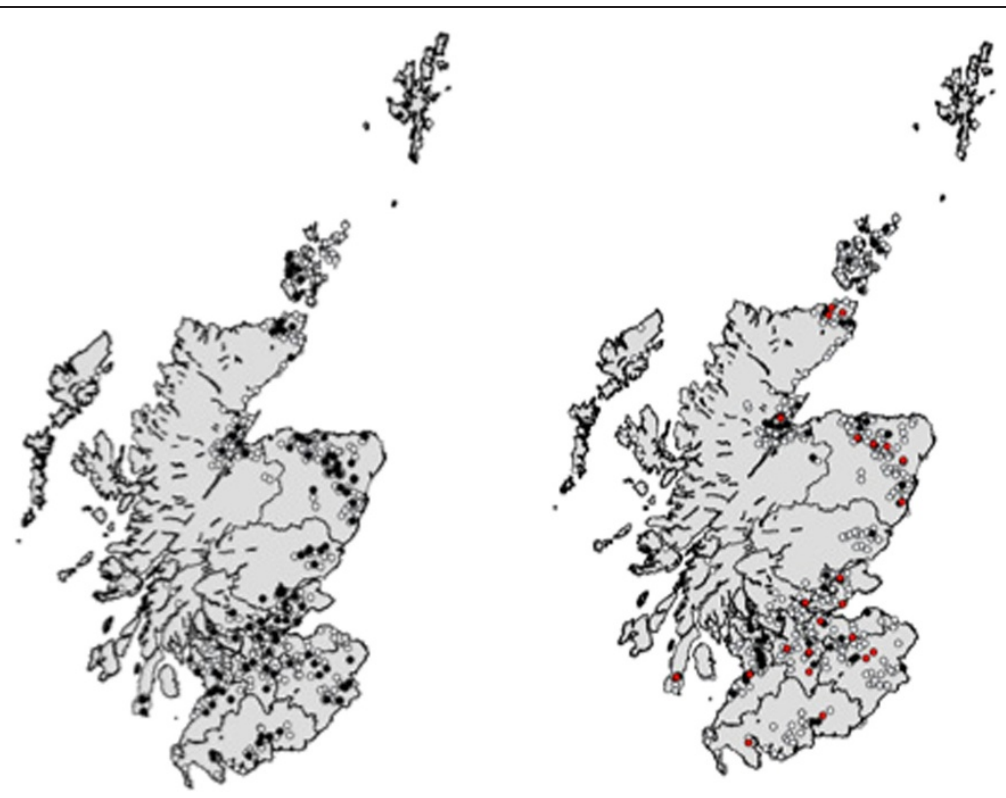

Figure 1 The geographical distribution of Scottish farms from both Survey 1 (SEERAD, left) and Survey 2 (IPRAVE, right) surveys ( $\mathbf{n}=\mathbf{4 4 7 )}$ ). Left: Survey 1 (SEERAD, 1998-2000). White circles represent E. coli O157 negative farms, black circles represent E. coli O157 positive farms. Right: Survey 2 (IPRAVE, 2002-2004). White circles represent 0157 negative farms, black circles represent E. coli O157 positive farms and red circles represent the 27 farms that were E. coli 0157 positive in both surveys. 
nature of the available information, no risk factors associated with feed, management or the presence of other livestock were identified in the final model. In addition there is no evidence that the previous status of the farm is important. This agrees with the conclusion of Zhang et al [6] which suggested that in a given year approximately $80 \%$ of the farms in Scotland will likely have cattle that are shedding E. coli O157. It also reaffirms the position of public health officials who have recommended that all farms in Scotland should be considered positive [8]. In keeping with a prior analysis of the Survey 2 (IPRAVE) data the risk factors identified included the size of the farm (total number of cattle) and recent movements (represented here by the number of cattle moved onto the farm within 8 weeks of sampling). The absolute odds ratio (SD) from the Zhang et al. [6] paper was only 1.297 (0.379). The addition of risk factors pertaining to local spread (local farm positive) increased the odds ratio (SD) of the overall risk model to 2.61 (0.79). Zhang et al. [6] found no evidence of local spread although this was not tested directly as we have done in this study. It may be the case, however, that local spread is only of increased risk within certain regions as suggested by the interaction of AHD and local farm positive. Farms in areas of North East, Highland and South West were significantly more at risk if a local farm was identified as positive. This may reflect differences in biosecurity within these regions. Although the importance of spatially variable factors such a distance between farms (neighbours can be further apart in some area than others) [25] and topography cannot be discounted.

Two important findings within this study are evidence of local spread and persistence. Indistinguishable PFGE profiles were present on local farms, suggesting local farm-to-farm spread of E. coli O157 strains, and on farms after a period of approximately 3 to 4 years, suggesting persistence. These results are of even greater importance as with only one colony per pat being analysed our results are likely to underestimate the number of common strains. In addition, we only considered strains with indistinguishable PFGE profiles, not variants differing by one or two bands by PFGE. Evidence of local spread of E. coli $\mathrm{O} 157$ has been reported previously, [26]. However, literature suggesting frequent transmission between farms is rare. This study adds weight to a growing body of evidence, concurring with the results of two North American studies by Wetzel et al [27] and Rosales-Castillo et al [28]. The former found genetically indistinguishable isolates on neighbouring Ohio dairy farms whilst the latter reported the spread of E. coli $\mathrm{O} 157$ isolates between Mexican cattle farms. The results of our molecular analysis however cannot determine whether the spread of strains between farms is direct, due to factors such as cattle or human movement, or whether the indistinguishable strains we found come from a pool of environmentally persisting strains that are spread indirectly between farms through the movement of contaminated feed or wildlife, for example wild birds [27].

The presence of E. coli $\mathrm{O} 157$ on the closest local farms (farm in sample cluster positive) is a plausible risk factor as farms traditionally do not operate in isolation and farm staff within a locality may well visit other farms with some regularity, as well as using shared resources such as feed delivery or milking trucks. E. coli O157 could be transmitted to a new farm either through exchange of animals or by being transmitted by humans via foot or vehicle. The farm management questionnaire used in both Survey 1 and 2 did not ask about contacts with other farms so this potential transmission network cannot be tested formally. However, Rosales-Castillo et al [28] identified milking and other staff movement as a risk factor for farm-to-farm transmission. An alternative hypothesis could be that the clustering implies proximity to a shared environmental reservoir. In either case, cluster positivity encompasses the spread of E. coli $\mathrm{O} 157$ strains to and from local farms or an environmental reservoir.

Persistence of E. coli O157 PFGE profiles has been reported in the literature but the majority of this research was conducted as longitudinal studies [13,15,17,18,23] or repeated cross sectional surveys [10] of the same cohorts. Within this research E. coli O157 strains were observed to persist across the two surveys ( $\mathrm{n}=12$, Figure 2) as well as on the same farm $(n=3)$ after $3-4$ years and turnover of cattle. This result suggests that there may be certain strains within Scotland that have found a niche within the cattle environment. This is of concern as there seems to be an association with the existence of persistent cattle strains and the strains that are observed in human E. coli O157 cases.

\section{Conclusions}

Our findings highlight the relevance of local spread and persistent strains of E. coli O157 on Scottish cattle farms. The significance of local spread within certain AHDs is suggestive of biosecurity and or spatial geographical differences within Scotland. Further research is being conducted to determine biosecurity throughout Scotland and the importance of local geography on pathogen spread. Results of these studies may shed some light on the results of this research. The observation that there may be persistent strains within Scotland that are contributing to the number of human cases reported warrants further investigation. This is currently being investigated in a Scottish research collaboration.

\section{Methods}

\section{Data sources}

Data from multiple sources were pooled for use in this study including the following: (1) the June 2003 Agricultural 


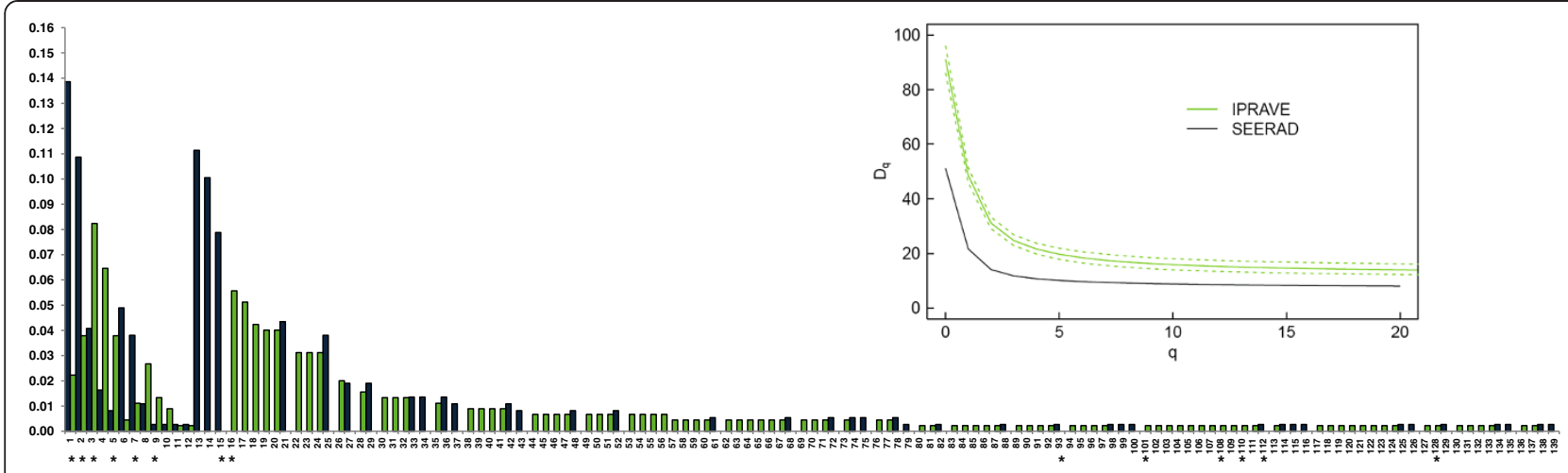

Figure 2 Distribution of the PFGE types observed in Survey 1 (SEERAD, black bars) and Survey 2 (IPRAVE, green bars). Distribution of PFGE types is organized so that the first 12 represent PFGE types that are common to both the field surveys. Asterisks represent PFGE types known to be present in human clinical samples for the same time frame. Inset graph is the diversity profiles for the Survey 2 (IPRAVE, green) and Survey 1 (SEERAD, black) surveys illustrating the significantly higher diversity of PFGE types observed in Survey 2 (IPRAVE). Dotted lines represent the confidence intervals for Survey 2 (IPRAVE) data generated by subsampling to the size of Survey 1 (SEERAD).

census of livestock premises combined with the department for Environment, Food and Rural Affairs (DEFRA) list of livestock premises; (2) the Cattle Tracing System (CTS), (3) Land Capability for Agriculture in Scotland; (4) E. coli nonO157 prevalence data; (5) E. coli O157 prevalence data, (6) E. coli O157 typing and PFGE data. Details on most of the data sources have been published previously however, each data set will be described briefly below.

(1) June 2003 Agricultural census data [29]. Among the original 50,266 farms in Scotland recorded in the census, 22,286 farms are provided with the numbers of animals but only 13,704 farms have cattle. Our system comprises these 13,704 cattle farms. The data include the Council-Parish-Holding number $(\mathrm{CPH})$, the X-Y coordinates of the farm-house, the area of the farm, and the numbers of cattle, sheep and pigs. The number of cattle on each farm is assumed constant at the number recorded in the census.

(2) Cattle Tracing System (CTS). The CTS is operated by DEFRA's British Cattle Movement Service [20,30]. In Scotland, during years 2002-2004 there were 252,496 movements among 11,464 of the cattle farms entered in the 2003 census database (the remainder are assumed not to have moved cattle to or received cattle from other farms). Movements outside Scotland and to/from abattoirs and markets are not considered here.

(3) Land Capability for Agriculture in Scotland (LCA). The LCA classification was developed by the Macaulay Institute to describe the agricultural potential of land based on the degree of limitation imposed by its biophysical properties. It is based primarily on climate, a number of soil properties, (for example depth and stoniness), wetness, erosion risk and slope. Also included are the overall pattern, i.e. variability, and, in one of the classes (Class 6), vegetation cover is also taken into account. The LCA is a seven class system where class 1 represents land that has the highest potential flexibility of use whereas class 7 land is of very limited agricultural use [21].

(4) E. coli O157 prevalence data. Between March 1998 and February 2004 two cross sectional surveys were conducted in Scotland. Data used in this study came from 447 farms sampled in both surveys (Figure 2). The first survey (Survey 1) was conducted by the Scottish Agricultural College through funding from the Scottish Executive Environment and Rural Affairs Department (SEERAD) from March 1998 to May 2000. The second survey (Survey 2) was carried out between February 2002 and February 2004, funded by the Wellcome Trust International Partnership Research Award in Veterinary Epidemiology (IPRAVE). The field sampling methodologies for both surveys have been described in previous literature $[7,12]$, however, a brief outline is given below. Further, farmers were asked to complete a farm management questionnaire from which much of the data about the farms were gathered. Prior to sampling, written consent was obtained from the farmers for participation in the study.

Both surveys preferentially sampled cattle groups composed only of store (i.e. weaned cattle before finishing for slaughter) or finishing cattle closest to sale or slaughter. If such groups did not exist, one or more mixed groups with store or finishing cattle closest to sale or slaughter were sampled. From each group fresh faecal pats were sampled. The number of pats tested in each group was determined from the number of cattle in the group using a prescribed sampling schedule. For Survey 1 , sufficient numbers of faecal pats were tested to ensure prospectively an $80 \%$ chance of sampling at least one positive pat if there was a shedding prevalence of at least $2 \%$ within the group [12]. Based on results from Survey 1 , in Survey 2, it was assumed that, on average, $8 \%$ of the animals in positive groups would be shedding, with shedding distributed as seen in Survey 1 [7]. For each 
group in Survey 2, sufficient fresh pat samples were taken to ensure prospectively a mean $90 \%$ probability of detecting shedding of $E$. coli $\mathrm{O} 157$ if at least one shedding animal was indeed present. Changes in sampling strategy between the two surveys had a negligible effect on the power to identify positive farms [7]. Instead of randomly sampling farms within each Animal Health District (AHD), Survey 2 used a stratified sampling plan derived from the Survey 1 cohort to select farms to sample [31]. Farms were selected randomly then the farms with closest Euclidean distances were sampled on the same or concurrent days, leading to clusters of 3 independent farms. The sampling of the same farms between the studies and the comparable methodology between the studies allow the use of these separate surveys as two cross-sectional time points for our analysis.

(5) E. coli non O157 prevalence data [32]. Collection of bovine isolates for the detection of E. coli O26, O103, O111 and O145 done in parallel with Survey 2 (20022004). Fecal samples were taken from 338 farms to test for non-O157 E. coli strains as described in [32].

(6) E. coli O157 typing data. Within 48 hours of sampling, one gram of faeces from each pat sample was tested for the presence of E. coli O157 through immuno-magnetic separation (IMS) and culturing as described in detail elsewhere [33]. Following IMS, one E. coli O157 isolate from each faecal sample was submitted to the Scottish E. coli O157/VTEC Reference Laboratory (SERL) for phage typing, and testing for the presence of genes encoding the virulence factors shigatoxin 1 (st $\left.x_{1}\right)$, shigatoxin $2\left(s t x_{2}\right)$ and intimin (eae) using multiplex PCR. PFGE analysis was conducted on E. coli $\mathrm{O} 157$ isolates from both surveys as described previously [33] as well as randomly selected isolates selected from human clinical samples from the same time frame. Briefly, isolates were digested with $50 \mathrm{U}$ of $\mathrm{XbaI}$ restriction enzyme, then subjected to PFGE using CHEF DRII apparatus (Bio-Rad laboratories, UK). Further analysis and categorization of PFGE results were conducted using Bionumerics 4.1 (Applied Maths, Belgium).

\section{Statistical analysis}

\section{Agreement between surveys}

The presence and absence of agreement between farm $E$. coli O157 status on farms in Survey 1 and Survey 2 was compared using McNemar test. McNemar's test assesses the significance of the difference between two correlated proportions. The analysis was performed using StatXact version 8 (Cytel Software Corp, Cambridge, MA, USA). The null hypothesis is that the proportion of farms with the characteristic (or event) is the same for Survey 1 and Survey 2.

\section{Risk factor analysis}

Data from all of the sources listed above was compiled and used as the basis of the risk factor analysis. Risk factors for the presence of E. coli O157 on a farm were analysed using logistic regression analysis (Proc Logistic, SAS Institute Inc., Cary, NC). Logistic regression analyses were carried out on a single variate basis initially. All the potential risk factors (Additional file 1: Table S1, $\mathrm{n}=49$ ) were examined. All variables with a $\mathrm{p}$ value of $<0.2$ were retained for multiple variate analysis. Region and season were forced into the model as design factors. Seasons were defined as winter, comprising December, January, and February; spring, comprising March, April and May; Summer, comprising June, July and August; and Autumn, comprising September, October and September. Six regions, based on Veterinary Animal Health Districts (AHDs) were defined: 1 = Islands; 2 = Highland; 3 = North East; $4=$ Central; $5=$ South East; $6=$ South West. A hierarchical forward selection and backward elimination approach with swapping (reassessment of previously included or excluded variables) were used. The change in the deviance of the model was monitored as an indicator of improved fit. Variables were added and removed based on significant improvement in the mean deviance after changes to the model. Two-way interactions were also tested in this manner. The inclusion of a random effects term for cluster did not improve the fit of the model significantly.

Model fit was assessed by fitting ROC curves to the final models and generating area under the curve (AUC) statistics for the models. The AUC can be considered to be a measure of the discriminatory power of the model [34]. A theoretically perfect model would have an AUC $=1$ while a model with no discriminatory power would have AUC $=0.5$. Thus using this scale, the AUC statistics of the models and hence the probability of the model being able to discern between a positive and negative E. coli $\mathrm{O} 157$ farm can be assessed and compared. For the final model, the Hosmer-Lemeshow goodness-of-fit statistic was computed [19].

To check for multicollinearity between factors in the final model, correlations were examined for binary and nominal variables. In addition, the stability of the model was checked by systematic removal of variables. Diagnostics were performed and plots of residuals were examined, confirming goodness of fit of the model. Odds ratios and their associated $95 \% \mathrm{CI}$ were estimated in the final model for factors statistically significantly associated with the presence of E. coli O157.

To compare the results of the statistical analysis in this study to those generated earlier by Zhang et al. [6] an overall odds ratio estimate that was developed for the earlier study was calculated. Values for the empirical estimate of the odds ratio were derived using the parameter estimates from the logistic regression model. These estimates were used in the statistical model to simulate binary response random variables for each farm (absence/presence 
of E. coli $\mathrm{O} 157$ on-farm). The predicted presences/absences were then related to the observed presences/absences, and aggregated over all farms, from which summaries an odds ratio was calculated by

$$
O R=\frac{F_{++} \times F_{--}}{F_{+-} \times F_{-+}}
$$

where $F_{++}$is the number of farms that are positive for both model prediction and observed data, and the meanings of $F_{--}, F_{+-}$and $F_{-+}$follow accordingly [6]. An odds ratio greater than 1 indicates that the model is more likely than not to predict the correct infection status of a farm. The larger the odds ratio, the stronger the predictive power of the model. This process was repeated to produce a distribution of odds-ratios which could then be summarised.

\section{Local spread and persistence}

Survey 2 sampling clusters in which $>1$ farms were positive were investigated to determine whether any identical E. coli $\mathrm{O} 157$ strains could be found on farms within the vicinity of each other (average distance $=5.96 \mathrm{~km}$ ). BioNumerics 4.1 software (Applied Maths, Belgium) was used to analyse the PFGE profiles. Dendrograms were generated using unweighted pair group method with arithmetic mean (UPGMA) with settings of $1.00 \%$ optimisation and $1.3 \%$ position tolerance and provided a visual representation of the relationships among isolates. Each unique PFGE profile was allocated a profile identifying code (1-139). For the purposes of our local spread analysis, isolates had to be indistinguishable (ie 100\% similar) for classification as locally spreading. Analysis determined whether sampling clusters were more likely to have the same PT / PFGE profile than random. To do this the clustered data were compared to 10,000 bootstrap samples of pairs of positive farms sampled at random.

Farms that were E. coli $\mathrm{O} 157$ positive in both Survey 1 and Survey $2(\mathrm{n}=27$, Table 1$)$ were analysed using the same dendrogram protocol. This analysis was conducted to identify any strains that were present in both Survey 1 and Survey 2. The criteria for being defined as the same strain was $100 \%$ similarity using the above dendrogram protocol. This is a conservative estimate and likely a lower estimate as it does not allow for common strain development.

\section{Ecological diversity of PFGE types}

Diversity of PFGE types in both surveys was examined using multiple diversity measures, related to Renyi's measures of generalized entrophy $[35,36]$ similar to the analysis done in Mather et al. [37]. The exponential of Renyi's entrophy measure gives an estimate of the effective number of species $D_{q}[38,39]$, with its single parameter $q$ determining the extent to which rare PFGE types (in this instance) contribute towards overall diversity (Equation 2). The following diversity indicies were calculated for both surveys: specie richness (SR), $\mathrm{D}_{0}$; Shannon Entrophy (SE), $\log \left(\mathrm{D}_{1}\right)$; Simpson diversity $(\mathrm{SD}), 1 / \mathrm{D}_{2}$; and Berger-Parker (BP), $1 / \mathrm{D}_{\infty}$

$$
\mathrm{D}_{\mathrm{q}}\left(\mathrm{p}_{1} \mathrm{Kp}_{\mathrm{s}}\right)= \begin{cases}{\left[\sum_{i=1}^{s} p_{i}^{q}\right]^{1 / 1-\mathrm{q}},} & \mathrm{q} \neq 1, \\ \prod_{i=1}^{s} p^{\mathrm{i}} p^{p \mathrm{i}}, & \mathrm{q}=1\end{cases}
$$

While it is trivial to say that values of $\mathrm{D}_{0}$ would inform on the total number of different PFGE types present in the survey data, values of $D_{\infty}$ would carry out some information on the number of PFGE types that seems to dominate the profiles observed.

To measure the diversity of each sample (Survey 1 and Survey 2), true abundances of the sample were derived directly from the count data $\left(\mathrm{p}_{\mathrm{j}}=\mathrm{n}_{\mathrm{j}} / \Sigma \mathrm{n}_{\mathrm{j}}\right)$, and the different diversities calculated from the abundance proportions. However, because sample diversity measures depend heavily on sample size [40], direct comparison was conducted by repeatedly subsampling the larger (Survey 2) sample to the size of the smaller (Survey 1) sample with replacement. The algorithm was applied 1000 times to generate 1000 different sub-dataset of similar size as Survey 1 and enabling the creation of confidence intervals around $\mathrm{D}_{\mathrm{q}}$ and, therefore, every every measure examined. Analysis was conducted in $\mathrm{R}$ version 3.0.2 (R Development Core Team, 2013) [41].

\section{Additional file}

Additional file 1: Table S1. List of all risk factors examined in this study The 49 variables are grouped according to the broad categories. C, this variable was treated as a categorical variable for statistical analyses; $Q$, this variable was treated as a quantitative variable for statistical analyses.

\section{Competing interests}

We declare that none of the authors at the time of the study or preparation of the paper have any competing interests that could influence or bias the content of this paper.

\section{Author's contributions}

$\mathrm{LJH}$ conducted the initial statistical analysis and wrote the initial manuscript. LV and DVH performed the PFGE analysis. GJG co-ordinated the field sampling. MCP and DM conducted field sampling. Gl helped with the statistical analysis of PFGE profiles. IJM provided the code to calculate the absolute odds ratio. TP generated the diversity profiles. LA performed the phage typing, LA and MH provided the clinical isolates for the study and ML provided information on human case data in Scotland. LM contributed to final manuscript. MEJW coordinated the epidemiological study and contributed to final manuscript. MECT created the dataset and performed the final statistical models and made the revisions required for submission of the final manuscript. All authors read and approved the final manuscript.

\section{Acknowledgements}

We thank Alastair Smith, Hazel Knight, Judith Evans and Geoff Foster for their work in the laboratory. Dr. Paul Bessel provided the LCA data for the farms in the study. Survey 2 was funded by the Wellcome Trust International Partnership Research Award in Veterinary Epidemiology (IPRAVE) project and The Scottish 
Executive Environment and Rural Affairs Department (SEERAD: Survey 1). SEERAD Census Division provided a randomised list of farms from the sampling frame. The CTS data and Census data extracts used in this study were provided through funding by the Centre of Expertise on Animal Disease outbreaks (EPIC) funded by the Scottish Govennment. Dr. Thibaud Porphyre is funded by the Centre of Expertise on Animal Disease outbreaks (EPIC) funded by the Scottish Govennment (Programmes of Research 2011-2016 no UEH/851111). Dr l. McKendrick and Dr. G. Innocent are partially funded by the Scottish Government's Rural and Environment Science and Analytical Services Division (RESAS). Dr. Louise Matthews is funded by SPASE (the Scottish Partnership for Animal Science Excellence and the Food Standards Agency (Scotland).

\section{Author details}

${ }^{1}$ Centre for Immunity, Infection and Evolution, University of Edinburgh, King's Buildings, Edinburgh, UK. ${ }^{2}$ Medical Laboratory Sciences, Kuwait University, Kuwait City, Kuwait. ${ }^{3}$ Biomathematics and Statistics Scotland (BIOSS), University of Edinburgh, King's Buildings, Edinburgh, UK. ${ }^{4} 31 \mathrm{~A}$ Arthur van Dycklaan, Tervuren 3080, Belgium. ${ }^{5}$ Institute of Comparative Medicine, Faculty of Veterinary Medicine, Bearsden, University of Glasgow, Glasgow, UK. ${ }^{6}$ Health Protection Scotland, Meridian Court, Cadogan St, Glasgow, UK. ${ }^{7}$ Scottish E. coli O157NTEC Reference Laboratory, Royal Infirmary, Edinburgh, UK. ${ }^{8}$ Institute of Biodiversity, Animal Health \& Comparative Medicine, College of Medical, Veterinary and Life Sciences, University of Glasgow, Bearsden

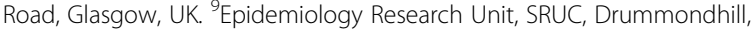
Stratherrick Road, Inverness, UK.

Received: 18 March 2013 Accepted: 26 March 2014 Published: 26 April 2014

\section{References}

1. Yim JY, Yoon J, Hovde CJ: A Brief Overview of Escherichia coli O157: H7 and its Plasmid O157. J Microbiol Biotechnol 2010, 20(1):5-14.

2. Chase-Topping ME, Gally D, Low C, Matthews L, Woolhouse MEJ: Supershedding and the link between human infection and livestock carriage of Escherichia coli 0157. Nat Rev Microbiol 2008, 6:904-912

3. Mead PS, Griffin PM: Escherichia coli O157:H7. Lancet 1998, 352:1207-1212.

4. Sakuma M, Urashima M, Okabe N: Verocytotoxin producing Escherichia coli, Japan, 1999-2004. Emerg Infect Dis 2006, 12:323-325.

5. Locking ME, Allison L, Smith-Palmer A, Rae L, Hanson M, Cowden J: Most farm related E. coli $\mathrm{O} 157$ outbreaks in Scotland occur on private farms, rather than open farms. [http://www.documents.hps.scot.nhs.uk/posters/2010/ farms-ecoli-scotland-private-open.pdf].

6. Zhang XS, Chase-Topping ME, McKendrick IJ, Savill NJ, Woolhouse MEJ: Spread of E. coli 0157 infection among Scottish cattle farms: stochastic models and model selection. Epidemics 2010, 2(1):11-20.

7. Pearce MC, Chase-Topping ME, McKendrick IJ, Mellor DJ, Locking ME, Allison L, Ternent HE, Matthews L, Knight HI, Smith AW, Synge BA, Reilly W, Low C, Reid SWJ, Gunn GJ, Woolhouse MEJ: Temporal and spatial patterns of bovine Escherichia coli 0157 prevalence and comparison of temporal changes in the patterns of phage types associated with bovine shedding and human E. coli 0157 cases in Scotland between 1998-2000 and 2002-2004. BMC microbiology 2009, 9:276-289.

8. Locking M, Cowden J: Escherichia coli 0157. Early clinical diagnosis and outbreak investigation are essential to prevent serious sequelae and further transmission. BMJ 2009, 339:817-819.

9. Hancock DD, Besser TE, Rice DH: Ecology of Escherichia coli O157:H7 in cattle and impact of management practices. In Escherichia coli O157:H7 and Other Shiga Toxin-Producing E. coli Strains. Edited by Kaper JB, O'Brien AD. Washington, DC: ASM Press; 1998:85-91.

10. Conedera G, Chapman PA, Marangon S, Tisato E, Dalvit P, Zuin A: A field survey of Escherichia coli 0157 ecology on a cattle farm in Italy. Int J Food Microbiol 2001, 66:85-93.

11. Robinson SE, Wright EJ, Hart CA, Bennett M, French NP: Intermitent and persistent shedding of Escherichia coli 0157 in cohorts of naturally infected calves. J App/ Microbiol 2004, 94:1045-1053.

12. Gunn GJ, McKendrick IJ, Ternent HE, Thomson-Carter F, Foster G, Synge BA: An investigation of factors associated with the prevalence of verocytotoxin producing Escherichia coli 0157 shedding in Scottish beef cattle. Vet journal 2007, 174(3):554-564
13. Rahn K, Renwick SA, Johnson RP, Wilson JB, Clarke RC, Alves D, McEwen S, Lior H, Spika J: Persistence of Escherichia coli 0157 in dairy cattle and the dairy farm environment. Epidemiol Infect 1997, 119:251-259.

14. Shere JA, Bartlett KJ, Kaspar CW: Longitudinal study of Escherichia coli 0157:H7 dissemination on four dairy farms in Wisconsin. Appl Environ Microbiol 1998, 64:1390-1399.

15. Liebana E, Smith RP, Lindsay E, McLaren I, Cassar C, Clifton-Hadley FA, Paiba GA: Genetic diversity among Escherichia coli 0157:H7 Isolates from bovines living on farms in England and Wales. J Clin Microbiol 2003, 41(8):3857-3860.

16. Renter DG, Sargeant JM, Oberst RD, Samadpour M: Diversity, frequency and persistence of Escherichia coli 0157 strains from range cattle environments. Appl Environ Microbiol 2003, 69:542-547.

17. LeJeune JT, Besser TE, Rice DH, Berg JL, Stilborn RP, Hancock DD: Longitudinal study of fecal shedding of Escherichia coli 0157:H7 in feedlot cattle: predominance and persistence of specific clonal types despite massive cattle population turnover. Appl Environ Microbiol 2004, 70(1):377-384

18. Liebana E, Smith RP, Batchelor M, McLaren I, Cassar C, Clifton-Hadley FA, Paiba GA: Persistence of Escherichia coli 0157:H7 Isolates on bovine farms in England and Wales. J Clin Microbiol 2005, 43(2):898-902.

19. Hosmer DW Lemeshow S: Applied Logistic Regression. 2nd edition. New Jersey: Wiley Publishing; 2000

20. BCMS: Cattle Tracing System (CTS) online. 2005. [www.bcms.gov.uk]

21. Bibby JS, Douglas HA, Thomasson AJ, Robertson JS: Land Capability for Agriculture. Soil Survey of Scotland Monograph. Aberdeen: The Macaulay Institute for Soil Research; 1991.

22. Cernicchiaro N, McEwen S, Harpster L, Homan HJ, Linz GM, Lejeune JT: Association of Wild Bird Density and Farm Management Factors with the Prevalence of E. coli 0157 in Dairy Herds in Ohio (2007-2009). Zoonoses and ic Health 2012, 59(5):320-329.

23. Carlson BA, Nightingale KK, Mason GL, Ruby JR, Choat WT, Loneragan GH, Smith GC, Sofos JN, Belk KE: Escherichia coli O157:H7 strains that persist in feedlot cattle are genetically related and demonstrate enhanced ability to adhere to intestinal epithelial cells. Appl Environ Microbiol 2009, 75(18):5927-5937.

24. Jeong KC, Hiki O, Kang MY, Park D, Kaspar CW: Prevalent and persistent Escherichia coli 0157:H7 strains on farms are selected by bovine passage. Vet Microbiol 2013, 162:912-920.

25. Flood JS, Porphyre T, Tildesley MJ, Woolhouse MEJ: The performance of approximations of farm contiguity compared to contiguity defined using detailed geographical information in two sample areas in Scotland: implications for foot-and-mouth disease modelling. BMC Vet Res 2013, 9:198.

26. Rice DH, McMenamin K, Pritchett LC, Hancock DD, Besser TE: Genetic subtyping of Escherichia coli 0157 isolates from 41 Pacific Northwest USA cattle farms. Epidemiol Infect 1999, 122(3):479-484.

27. Wetzel AN, Lejeune JT: Clonal Dissemination of Escherichia coli O157: H7 Subtypes among Dairy Farms in Northeast Ohio. Appl Environ Microbiol 2006, 72(4):2621-2626.

28. Rosales-Castillo JA, Vázquez-Garcidueñas M, Alvarez-Hernández H, ChassinNoria O, Varela-Murillo Al, Zavala-Páramo M, Cano-Camacho H, VázquezMarrufo G: Genetic diversity and population structure of Escherichia coli from neighboring small-scale dairy farms. J Microbiol 2011, 49(5):693-702

29. Scottish Agricultural Census. 2005. [http://www.scotland.gov.uk/Resource/ Doc/47210/0017481.pdf].

30. Mitchell A, Bourn D, Mawdsley J, Wint W, Clifton-Hadley R, Gilbert M: Characteristics of cattle movements in Britain - an analysis of records from the Cattle Tracing System. Anim Sci 2005, 2005(80):265-273.

31. Halliday JEB, Chase-Topping M, Pearce MC, McKendrick IJ, Allison LJ, Fenlon D, Low C, Mellor DJ, Gunn GJ, Woolhouse MEJ: Herd-level risk factors associated with the presence of Phage type 21/28 E. coli 0157 on Scottish cattle farms. BMC Microbiol 2006, 6:99.

32. Pearce MC, Evans J, McKendrick IJ, Smith AW, Knight HI, Mellor DJ, Woolhouse MEJ, Gunn GJ, Low JC: Prevalence and virulence factors of Escherichia coli serogroups $026,0103,0111$ and 0145 shed by cattle in Scotland. Appl Environ Microbiol 2006, 72(1):653-659.

33. Vali L, Hamouda A, Pearce MC, Knight HI, Evans J, Amyes SGB: Detection of genetic diversity by pulsed-field gel electrophoresis among Escherichia coli $\mathrm{O} 157$ isolated from bovine faecal samples by immunomagnetic separation technique. Lett Appl Microbiol 2007, 44(1):19-23. 
34. Cook NR: Use and misuse of the receiver operating characteristic curve in risk prediction. Circulation 2007, 115(7):928-935.

35. Renyi A: On Measures of Information and Entrophy. In Proc. $4^{\text {th }}$ Berkeley Symp. On Mathematics, Statistics and Probabilty. Berkeley, CA: University of California Press; 1961:547-561.

36. Hill MO: Diversity and evenness - unifying notation and its consequences. Ecology 1973, 54:427-432.

37. Mather AE, Matthews L, Mellor DJ, Reeve R, Denwood MJ, Boerlin P, Reid-Smith RJ, Brown DJ, Coia JE, Browning LM, Haydon DT, Reid SWJ: An ecological approach to assessing the epidemiology of antimicrobial resistance in animal and human populations. Proc Biol Sci 2012, 279:1630-1639.

38. Jost L: Entropy and diversity. Oikos 2006, 113:363-375.

39. Leinster T, Cobbald CA: Measuring diversity: the importance of species similarity. Ecology 2012, 93(3):477-489.

40. Saunders H: Marine benthic diversity: a comparative study. Am Nat 1968 , 102:243-282.

41. R Core Team: R: A Language and Environment for Statistical Computing. Vienna, Austria: R Foundation for Statistical Computing; 2013. URL http:// www.R-project.org/.

doi:10.1186/1746-6148-10-95

Cite this article as: Herbert et al: E. coli $\mathrm{O} 157$ on Scottish cattle farms: Evidence of local spread and persistence using repeat cross-sectional data. BMC Veterinary Research 2014 10:95.

\section{Submit your next manuscript to BioMed Central and take full advantage of:}

- Convenient online submission

- Thorough peer review

- No space constraints or color figure charges

- Immediate publication on acceptance

- Inclusion in PubMed, CAS, Scopus and Google Scholar

- Research which is freely available for redistribution 